\title{
About ways of psyhoemotional status regulation of minigolf players
}

Keywords: golf, mini golf, sports, psychological readiness, functional music, essential odorants, functional status

\section{Opinion}

Various ways of regulation of the Psyhoemotional status of young players in mini golf are considered. Questions of application of the functional music and inhalations by essential oils are discussed. It is shown that listening of the functional music at lento-andante rate before a game reduces the Psyhoemotional tension of players. Besides such musical influences can be used for change of swing rate. Musical influences at the speed corresponding to the individual frequency of characteristic oscillating fluctuations of the top extremities and a club and torsional oscillations of a trunk increase game actions stability. Inhalations by various essential oils also have various effects on a psychoemotional and functional status of players. It is established that inhalations by an certain structure odorant statistically are authentically reduced by Kerdo's index of players by 3 times. At an assessment of influence of essential odorants on game results the greatest influence of inhalation by placebo mix on improvement of results is revealed. It is supposed that decrease of exaltation of players is caused not so much by chemical composition of the inhaled odorant, and the reflex decrease in a beat frequency of heart caused by deep respiration at inhalation.

The assessment and regulation of the functional athletes status when performing competitive exercises is one of the decisive factors defining a wrestling outcome in many sports. The functional status of an athlete organism is directly bound to his psychoemotional states - conditions of a stress, optimum readiness and frustration. ${ }^{1,2}$ At the same time changes of the functional state lead to change of a mental condition of the athlete, and, on the contrary, lead changes of a psychoemotional state to change of the functional state. Especially sharply the problem is shown at stages of the highest sports skill and in sports, the bound to preferred manifestations of accuracy, and, therefore, coordination abilities at multiple implementation of the same motive acts. Examples of such sports: firing, golf, mini golf, darts, curling, bowling, etc. In all these sports the result, eventually, is defined by abilities of athletes to dose the movements on amplitude (effort) and the direction of their commission.

Regulation of mental states in the conditions of competitions in the sports stated above, the bound to manifestation of target accuracy of movements, is usually bound to application of techniques of a psychomuscle, mental and autogenic regulation. ${ }^{1,3}$ These techniques, in most cases, are directed to decrease in exaltation of athletes. Except these ways of athletes status regulation and increase in effectiveness various ways and their combinations based on physiological reflexes are applied: breath holdings, relaxation of facial muscles, deep respiration, the arbitrariest synkineses, etc. ${ }^{3}$

All ways of regulation of the functional and mental states, eventually, are carried out in three ways: passive or active or their combination. The passive way is carried out at the expense of characteristic informational metabolic cost of the athlete: by strong-
Volume I Issue I - 2017

\author{
Aleksey Korolkov,' Evgeny Lysov, ${ }^{2}$ Oksana \\ Frizen $^{2}$ \\ 'Pedagogical Institute of Physical Culture and Sport, Moscow \\ City Pedagogical University, Russia \\ ${ }^{2}$ Samara Regional Golf Development Federation, Russia
}

Correspondence: Aleksey Korolkov, Moscow City Pedagogical University, Russia, Tel +7 (495) 319-42-45, Email Korolkovan@mgpu.ru

Received: May 13, 2017 | Published: May 17, 2017

willed efforts, self-massage, sensor isolation, visualization, etc. Techniques of a psychomuscle, mental and autogenic regulation belong to this way. The active way of a status regulation provides any external influences in the form of tactile, acoustic, thermal, mechanical, odorantical, visual and biochemical influences. It is possible to refer acupuncture, taping, use of the warming ointments, hearing of musical compositions, inhalations, the use of various substances, etc. to the active ways of status regulation of the external influences. Some types of these influences are forbidden to use by the World Anti-Doping Agency.

The purpose of our work - to estimate a possibility of the active mode regulation of a psychoemotional athletes status based on application of the functional music and inhalations by essential oils.

For this purpose 12 young minigolf players were examined in January-March, 2017. The research consisted in comparison of physiological indexes of athletes and their results of a game before and after various affects. Also influence exhaustion on the functional players status and sexual and age preferences was estimated at perception of various odorants. Odorants represented $10 \%$ of mix of essential oils of a bergamot, ylang-ylang, lemon, mint, sage muscat, basilic, geraniums, lavenders, rosemary and an eucalyptus in different combinations in oil of grape seed. Three mixes running down, by placebo and activating were made. ${ }^{3}$ The functional music represented expressly created musical composition with rate of 54 min.-1 (Popov I. A., 2017).

As indexes the arterial pressure and heart rate in which sizes then Kerdo's index paid off were fixed. In total 13520 measurements were taken. Results were processed with use of a package of the statistical programs Stadia 8.0. As a result of the conducted researches sexual and age distinctions in olfactory preferences of essential odorants were established, influence of inhalations of essential odorants and exhaustion on the functional condition of players in minigolf is defined, influence of inhalations of essential odorants on sports results of players is established.

In particular significant sexual distinctions in preferences of odorants are established statistically: young men give preferences 
to an activating odorant, and girls prefer an odorant with less sharp neutral aroma of Placebo. As a result of statistical data processing of the experimental inspections statistically significant age distinctions in preference of aromas are not revealed. However the preference tendency by teenagers of less pungent smells in comparison with more adult athletes who also prefer an activating odorant is on average revealed. This conclusion demands further researches on large volumes of selections of examinees.

Analogies between features of color and olfactory preferences which can be used as for an assessment of the current mental and functional state of athletes, and for regulation of these states are described. At researches of influence of odorants on the athletes functional status is established that all odorants, including placebo lead to decrease in processes of exaltation in activity of the autonomic nervous system. At the same time the greatest effect of decrease in exaltation is caused by inhalation by an activating odorant which action is comparable with influence of exhaustion. Besides various individual distinctions in operation of various odorants on regulation of the functional indexes are revealed. It is supposed what changes in the functional state can be defined not only by biochemical influence of odorants, but also reflex reactions of an organism to the deep respiration which is carried out during three-minute intervals. It is also established that the greatest influence on decrease of an Kerdo's indexf, in comparison with inhalations, causes the exhaustion of players caused by multiple repetition of the monotonic same game actions within several clocks.

Regarding influence of odorants on sporting achievements it is established that on average improvement of results happens after inhalations by activating and placebo essential mix. In this regard it is supposed that this effect can be also caused by reflex reactions to deep respiration, and not just chemical composition of the inhaled odorants. As well as at a research of changes of the functional state various individual reactions of athletes in the form of sports results on incentives in the form of inhalations are established by various odorants.

It is established that the effect of influence of the functional music is comparable with effect of influence of an activating odorant and exhaustion. Significant distinctions in medians of Kerdo's index by Vilkokson's criterion for pair data at the level of a statistical significance are revealed statistically $p=0.05$. Besides, the effect of decrease in rate of game actions and increase of their stability after musical influence is established. In this direction it is possible to refer an opportunity research to prospects of further researches decrease of the monotonic exhaustion in minigolf by means of musical influences of different speed and the frequenciest structure, the choice of individual rate of a game for the different lines, applications of different structure essential odorants, influence on the functional condition of separate essential oils, but not their combinations. Perspective the repeated specifying researches of essential oils application and the functional music and their influence on sporting achievements, a mental and functional players status on large volumes of selections of examinees of one age and a floor are also represented.

\section{Acknowledgements}

Authors express special gratitude to the prof. N. N. Sentyabrev (The Volgograd State Academy of physical culture) for the valuable advice, remarks and recommendations stated when carrying out this work and also to the composer I. A. Popov (Allovers, Samara) who created the functional melody used as an acoustic stimulus. We thank our friend, the player in mini golf Astra Miglane Stanwyck for critical remarks and editorial editing of this article.

\section{Conflict of interest}

Authors declares there is no conflict of interest in publishing the article.

\section{References}

1. Korolkov AN. Minigolf: theoretical and methodical bases of sports preparation: monograph. Editus, Moscow, Russia; 2015. p. 264.

2. Lysov EA, Frizen OI, Pravdin DV, et al. Experience of carrying out and the organization of classes in sports minigolf during the express preparatory period. Izvestia TulGu. 2017;1:153-161.

3. Sentyabrev NN, Karaulov VV, Kaidalin VS, et al. Essential oils in sports practice: monograph. VGAFK. Volgograd, Russia; 2009. p.138. 